\title{
Reflets
}

Revue d'intervention sociale et communautaire

\section{Entre valeurs humanistes et modèles d'intervention : réflexions théoriques sur le sentiment de non-reconnaissance des travailleurs sociaux}

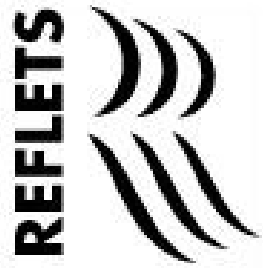

\section{Isabelle Chouinard}

Volume 19, numéro 2, automne 2013

La souffrance psychique et morale au travail : enjeux pour les professionnels du secteur de la santé et des services sociaux

URI : https://id.erudit.org/iderudit/1021184ar

DOI : https://doi.org/10.7202/1021184ar

Aller au sommaire du numéro

Éditeur(s)

Reflets, Revue d'intervention sociale et communautaire

ISSN

1203-4576 (imprimé)

1712-8498 (numérique)

Découvrir la revue

Citer cet article

Chouinard, I. (2013). Entre valeurs humanistes et modèles d'intervention : réflexions théoriques sur le sentiment de non-reconnaissance des travailleurs sociaux. Reflets, 19(2), 164-179. https://doi.org/10.7202/1021184ar

\section{Résumé de l'article}

L'identité professionnelle constitue une source d'enjeux constants en travail social. Si les services sociaux tendent à être méconnus, voire dévalorisés, par la population (Stephensen, et collab., 2000), un sentiment de non-reconnaissance est fréquemment ressenti chez les travailleurs sociaux, allant jusqu'à provoquer un haut taux de détresse et de retrait au travail (Pelchat, et collab. 2004). Par-delà les contraintes externes qui pèsent sur elle, l’identité professionnelle relève néanmoins d'un enjeu plus fondamental au sein de la profession. Sa professionnalité se fondant sur une dimension relationnelle hautement abstraite, symbolique et complexe, la pratique professionnelle et les contributions propres au travail social demeurent difficilement compréhensibles pour plusieurs. Cet article s'attarde à problématiser la dimension relationnelle du travail social et à proposer quelques pistes de réflexion pouvant mieux cerner l'origine des difficultés qui y sont reliées.
Tous droits réservés @ Reflets, Revue d’intervention sociale et communautaire, 2013
Ce document est protégé par la loi sur le droit d'auteur. L'utilisation des services d'Érudit (y compris la reproduction) est assujettie à sa politique d'utilisation que vous pouvez consulter en ligne. 


\section{Entre valeurs humanistes et modèles d'intervention : réflexions théoriques sur le sentiment de non-reconnaissance des travailleurs sociaux}

Isabelle Chouinard

Professeure en travail social

Département des sciences du développement humain et social Directrice de l'Équipe de recherche et d'analyse des pratiques professionnelles (ERAPP)

Université du Québec en Abitibi-Témiscamingue

Centre d'études supérieures Lucien-Cliche

\section{Résumé}

L'identité professionnelle constitue une source d'enjeux constants en travail social. Si les services sociaux tendent à être méconnus, voire dévalorisés, par la population (Stephensen, et collab., 2000), un sentiment de non-reconnaissance est fréquemment ressenti chez les travailleurs sociaux, allant jusqu'à provoquer un haut taux de détresse et de retrait au travail (Pelchat, et collab., 2004). Par-delà les contraintes externes qui pèsent sur elle, l'identité professionnelle relève néanmoins d'un enjeu plus fondamental au sein de la profession. Sa professionnalité se fondant sur une dimension relationnelle hautement abstraite, symbolique et complexe, la pratique professionnelle et les contributions propres au travail social demeurent difficilement compréhensibles pour 
plusieurs. Cet article s'attarde à problématiser la dimension relationnelle du travail social et à proposer quelques pistes de réflexion pouvant mieux cerner l'origine des difficultés qui y sont reliées.

\section{Introduction}

"D'ailleurs, le travail social a dès sa naissance mis en exergue la nécessité de l'établissement d'une relation avec un usager pour susciter sa coopération. "
La question de l'identité professionnelle des travailleuses sociales et des travailleurs sociaux est l'objet de débats et d'enjeux récurrents au sein de la profession. Déjà en 1960, la Corporation des travailleurs sociaux du Québec considérait que l'identité professionnelle de ses membres devait figurer parmi ses champs d'action prioritaires (Carey-Bélanger, 1979). Aujourd'hui encore, le phénomène identitaire demeure une préoccupation centrale au sein du travail social. À titre d'illustration, deux des trois buts stratégiques découlant des orientations privilégiées par l'Association canadienne des travailleuses et travailleurs sociaux (ACTS) pour les années 2012 à 2015 touchent de très près la question de l'identité professionnelle (ACTS, 2012). La promotion et le renforcement de la profession y sont préconisés afin d'améliorer la considération à l'égard du travail social au pays.

Si une attention soutenue est portée à la professionnalité du travail social, c'est qu'un malaise sous-tend sa pratique même, lequel peut être attribuable au caractère éminemment relationnel de toute intervention sociale. C'est en réalité sa nature si complexe et si souvent abstraite qui fait qu'elle apparaît a priori insaisissable de manière rationnelle. Pourtant, aussi difficilement accessible qu'elle puisse paraitre, l'importance de la relation au cœur de la pratique du travail social va bien au-delà de la seule facilitation de son intervention : c'est dans et par elle que s'incarne la spécificité de son action professionnelle (Autès, 1998). En ce sens, elle constitue le pivot de la professionnalité du travail social (Couturier et Chouinard, 2008).

D'ailleurs, le travail social a dès sa naissance mis en exergue la nécessité de l'établissement d'une relation avec un usager pour susciter sa coopération. Par leurs "visites amicales ", les premières auxiliaires sociales ont même été considérées comme les expertes 
de la relation; cette dernière étant nécessaire à la coopération de l'usager. La nature de la relation semble cependant toujours apparue d'une telle complexité que son analyse - et par voie de conséquence, sa capacité à se dire et à s'exposer clairement — pose depuis longtemps des défis importants pour de nombreux acteurs du travail social. La profession souffre d'un sentiment de nonreconnaissance par la population (Stephensen, et collab., 2000) et par le champ des professions humaines et sociales en général. Une difficulté à concevoir et à énoncer concrètement sa pratique est rencontrée chez plusieurs acteurs, au point de provoquer chez les travailleurs sociaux un taux élevé de détresse et de retrait au travail (Pelchat, et collab., 2004).

Si le cœur de la profession se joue autour d'une cruciale dimension relationnelle, il demeure que la source des difficultés identitaires reste encore incertaine. C'est dans la perspective de jeter un peu de lumière sur ce côté obscur de la relation que nous retournerons, le temps de quelques réflexions, aux origines du travail social. Ce détour théorique se veut une occasion d'associer certaines des tensions actuelles vécues en travail social à des tendances parfois contradictoires qui se sont très tôt imposées dans sa genèse.

Plusieurs ont écrit sur la question des fondements du travail social (Garnier, 2000; Ion, 2005) et sur celle de son évolution à travers des époques (Groulx, 1993; Mayer, 2002; Hurtubise et Deslauriers, 2003). La genèse de la profession, déjà connue par ailleurs, ne sera donc pas reprise ici. En réalité, si les fondements sont abordés, c'est dans une tentative de mieux comprendre le phénomène de l'identité professionnelle incertaine des

"Si notre propos reste principalement centré sur le travail social québécois, il n'en demeure pas moins qu'une réalité analogue s'est vécue dans l'ensemble $d u$ pays. » travailleurs sociaux (Vilbrod, 2003). Nous construirons pour ce faire notre réflexion autour de deux moments charnières pour la constitution du travail social au Québec : sa naissance, officialisée par l'adoption de la première Loi sur l'assistance publique de 1921, et sa professionnalisation dans les années 1950.

Si notre propos reste principalement centré sur le travail social québécois, il n'en demeure pas moins qu'une réalité analogue s'est vécue dans l'ensemble du pays. Le processus de professionnalisation s'est même accompli de manière plus accélérée dans les autres 
provinces. Au cours de la décennie 1940-1950, les avancées sociales au pays sont significatives, notamment en ce qui concerne le développement de lois sociales. Les nombreux progrès sociaux du côté fédéral ne trouveront écho au Québec que dix ans plus tard. En raison de la forte présence de l'Église et d'une vague politique conservatrice dans la province, le Québec résistera à s'engager, contrairement au Canada anglais, "dans la voie de l'intervention étatique et du professionnalisme social laïque " (Mayer, 2002, p. 120). Malgré tout, la question d'une meilleure définition du travail social à travers des époques reste une préoccupation centrale dans l'ensemble du Canada (Jennissen et Lundy, s.d.).

Après avoir abordé la question de la professionnalité du travail social, quelques caractéristiques spécifiques de la relation en travail social seront dégagées. Nous exposerons ensuite les diverses tensions auxquelles le travail social a fait face dans sa constitution en tant que profession et qui influent toujours sur sa capacité à se penser et à se décrire comme pratique professionnelle.

\section{La professionnalité du travail social}

La professionnalité des métiers comportant une dimension relationnelle se justifie de façon générale selon deux principaux axes sémantiques (Couturier et Chouinard, 2008; Du Ranquet, 1991; Soulet, 1997). Tel que l'illustre le schéma suivant, la définition de leur activité professionnelle est structurée selon un rapport entre, d'une part, la technique, qui signifie la maîtrise de savoirs et de méthodes (Couturier et Chouinard, 2008) et qui renvoie dès lors à ce qui est maitrisable, communicable sous forme de règles (Soulet, 1997) et, d'autre part, le relationnel, c'est-à-dire la nécessité d'une composante transactionnelle essentielle à la réalisation de l'action (Couturier et Chouinard, 2008). Ce second axe sémantique implique une part d'indétermination qui échappe aux règles (Soulet, 1997) et qui rend difficile son énonciation en des termes généraux. 
Figure 1 - La professionnalité des métier relationnels

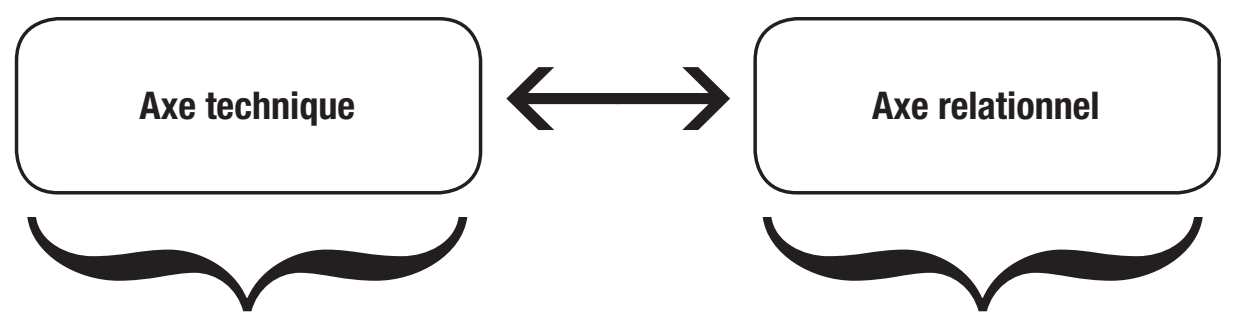

Maîtrise de savoirs et de méthodes

(Couturier et Chouinard, 2008)

Pratique communicable sous forme de règles

(Soulet, 1997)
Composante transactionnelle essentielle à la réalisation de l'action

(Couturier et Chouinard, 2008)

\section{Part d'indétermination}

(Soulet, 1997)

En raison de ses caractéristiques particulières, il appert que la professionnalité du travail social ne peut se justifier selon le seul axe technique. La nature symbolique du service rendu (Autès, 1998), les savoir-faire articulant dimensions psychoaffective et narrative (Ion et Ravon, 2005) mobilisés par les travailleurs sociaux, ainsi que l'absence d'un matériel tangible avec lequel intervenir (Soulet, 1997) sont autant de facteurs qui complexifient l'appréhension concrète du travail réalisé par les travailleurs sociaux. Une telle forme de travail rend en outre quasi impossible l'évaluation des productions concrètes du travail social. L'évaluation porte en fait le plus souvent sur la reconfiguration de l'expérience sociale des usagers (Giuliani, 2007). L'ergonome De Montmollin (1986) illustre bien cette difficulté vécue par les professionnels du travail social lorsqu'il avance (p. 32-33) qu'il existe

des tâches sans production, et même sans objectifs définissables. Des tâches sans critères évidents de réussite ou, ce qui revient au même, des tâches dont les productions sont si secrètes 
"Supprimer le travailleur social, la société continue apparemment comme avant. Tout est dans cet « apparemment» bien entendu.» ou si lointaines qu'elles échappent à l'analyse. Exemple extrême, mais réel, la plupart des travailleurs sociaux [...]. Supprimer le travailleur social, la société continue apparemment comme avant. Tout est dans cet "apparemment " bien entendu. Mais travailler uniquement à partir d'une certitude a priori, ou d'une foi, n'est pas une condition de travail souhaitable. Le statut psychologique des travailleurs sociaux, qui passent leur temps à s'interroger sur leur identité (en fait, leur production), n'est pas toujours enviable.

Ces propriétés relatives à la pratique du travail social font donc que la professionnalité de ce groupe s'articule principalement autour de l'axe relationnel. Dès lors qu'un groupe professionnel fonde son identité à partir de cet axe, trois principes axiomatiques viennent encadrer leur professionnalité :

Principe 1 : Les pratiques dans les métiers relationnels sont insécables des situations dans lesquelles elles se réalisent et sont donc inappréhendables par la seule raison objectivante.

Principe 2 : Bien qu'a priori langagières, ces pratiques relationnelles se caractérisent par une indicibilité fondamentale et donc par une indiscutabilité fondamentale dans l'espace public.

Principe 3 : Puisque le cœur de l'action est imperceptible par la seule raison et indicible à autrui, la formation doit porter a priori sur l'éthos professionnel comme principe directeur de l'agir professionnel plutôt que sur une forme ou l'autre de didactique des savoirs professionnels.

(Couturier et Chouinard, 2008, p. 214)

Suivant ces principes, le travail social peut être considéré comme une figure typique des métiers relationnels, parce qu'il 
"...la difficulté à exprimer le coeur du travail social est attribuable à son caractère complexe et multifocus. " est parmi les groupes professionnels dont les technologies sont les plus discursives et psychoaffectives qui soient. Pour plusieurs, la difficulté à exprimer le cœur du travail social est attribuable à son caractère complexe et multifocus. La question de la complexité du travail social est d'ailleurs fréquemment associée au fait qu'il

s'agit d'un travail «sur l'humain ", il n'existe pas de science éducative absolue, pas de connaissances sûres, pas de nosologie précise. Les connaissances du domaine sont en constante évolution, et il s'agit donc de travailler dans l'incertain et d'agir à la marge d'un système humain complexe. (Villate, Teiger et Caroly-Flageul, 2004, p. 594)

Pourtant, aussi complexe soit-elle, la profession est confrontée à l'obligation de rendre des comptes et de soumettre sa pratique à une évaluation concrète. Cela se révèle d'autant plus pénible pour les travailleurs sociaux qu'ils font face à un paradoxe, celui « du devoir dire et du devoir ne pas enfermer " (Soulet, 1997, p. 10) qui les contraint «à osciller entre la révélation de l'indicible et la monstration d'un espace irréductible à l'énonciation" (p. 18).

Dès lors qu'il s'agit de légitimation de leur pratique professionnelle, les arguments utilisés par bon nombre de travailleurs sociaux consistent dans l'adhésion à l'éthos de la profession, basé sur ses valeurs et ses finalités générales, ou encore dans la manifestation d'un savoir-faire résultant d'une application de modèles et de techniques d'intervention propres au travail social. L'absence de prise " concrète " sur la relation, sa difficulté à s'extraire et à se penser hors du champ de l'intervention in situ, oblige à justifier sa pratique par une croyance dans les valeurs humanistes et sociales promues par la profession ou encore par la maîtrise d'un processus d'intervention spécifique au travail social. Pour Zúñiga (1993), les travailleurs sociaux tendent à légitimer leurs interventions à partir d'arguments empruntés soit à la science (la Raison), soit aux priorités ministérielles (l'État) ou encore aux demandes faites par les usagers (l'Individu). Or, ces arguments ne rendent pas justice au cœur de la pratique professionnelle des travailleurs sociaux, aux savoirs qui y sont imbriqués et qui témoignent de sa spécificité, 
"Quels sont donc les savoirs propres au travail social? Le travail social peut-il prétendre accéder au statut de discipline scientifique?" puisque ceux-ci demeurent «dans la périphérie de la vitalité des pratiques » (Zúñiga, 1993, p. 35).

Quels sont donc les savoirs propres au travail social? Le travail social peut-il prétendre accéder au statut de discipline scientifique? Si dans le champ de l'éducation, philosophes, éducateurs, sociologues ou psychologues ont depuis longtemps réfléchi à la question du savoir, de sa transmission et de son acquisition, le développement de théories sur le travail social est relativement récent. Connu avant tout comme une discipline pratique dont les formes se modifient selon les transformations socio-politicoéconomiques, le travail social n'a été reconnu comme profession au Québec qu'à la fin de la décennie 1950. Dans ses efforts pour s'émanciper de son caractère vocationnel d'origine et se faire reconnaitre comme une véritable profession, le travail social a tenté de se constituer en discipline scientifique en se composant un corpus de connaissances qui lui est propre. Suivant cette logique, ce processus aurait en principe dû favoriser une identité professionnelle solide chez les acteurs. Pour quelles raisons alors le phénomène identitaire des travailleurs sociaux se trouve-t-il toujours au cœur des préoccupations à l'heure actuelle? De quels contextes cette problématique récurrente a-t-elle pu émerger? C'est ce à quoi nous tenterons de réfléchir en proposant quelques pistes d'éclaircissement.

\section{Les fondements du travail social ou la difficile articulation entre vocation et profession}

Afin de camper la réflexion, rappelons seulement que les organismes de charité et de bienfaisance ont influencé les premières formes d'intervention sociale individuelle sur la base d'un ensemble de valeurs liées à la morale religieuse catholique dominante de l'époque, tandis que le processus de professionnalisation du travail social a pour sa part fourni des assises théoriques aux problèmes sociaux et aux méthodes de pratique (Groulx, 1993). 
"Les approches d'intervention individuelle préconisées par les cuvres de charité, dont la plus populaire est le casework (Richmond, 1917), se sont donc imposées dès le départ au sein de la profession. »
En réalité, l'ensemble du travail social canadien a connu ces moments charnières. Partout au pays, la profession tire ses origines de la charité chrétienne (Jennissen et Lundy, s.d.) plaçant du coup l'accent sur les dimensions individuelles des problèmes, plutôt que sociales. Les approches d'intervention individuelle préconisées par les œuvres de charité, dont la plus populaire est le casework (Richmond, 1917), se sont donc imposées dès le départ au sein de la profession. En fait,l'influence de cette méthode est telle qu'elle sera enseignée dans les universités jusqu'en 1950. Les pratiques d'action communautaires ne viendront que plus tard enrichir le travail social, lors de la décennie suivant la Première Guerre mondiale où le pays connaitra une montée de formes d'activisme social (Jennissen et Lundy, s.d.).Au Québec, c'est en 1965 que des pratiques d'animation sociale auprès de groupes issus de classes populaires, créées entre autres pour s'opposer à des pratiques jugées trop individuelles (prédominance du casework) et trop préoccupées par des questions de reconnaissance professionnelle, verront le jour (Mayer, 2002). Des pratiques novatrices d'organisation communautaire seront dès lors mises en place.

La professionnalisation du travail social canadien se réalisera donc par un long processus qui comprendra différentes démarches d'émancipation à l'égard des traditions britanniques et étatsuniennes, dont il subira l'influence, tant sur le plan de la pratique que sur celui de la formation. Les inspirations venues des États-Unis sont par ailleurs telles que l'organisme américain Council of social work education a accrédité les formations canadiennes en travail social jusqu'au début des années 1970, ralentissant du coup le processus d'autonomisation de la profession au pays (Jennissen et Lundy, s.d.). Bref, la première tendance, chrétienne et individuelle, conservera un long moment sa forte connotation axiologique, surtout au Québec en raison de la vague conservatrice qui y prévaut jusque dans les années 1960. Cette influence politique a largement favorisé l'émergence d'un travail social de nature vocationnelle et confessionnelle dans la province (Groulx, 1993; Lecomte, 2000). On y décrivait le travail social comme

une profession qui ne relève ni du nursing, ni de l'enquête, ni de la distribution de secours. 
"...des

caractéristiques

individuelles,

généralement

attribuées au sexe

féminin, étaient

recherchées chez les

candidates..."
Cependant, le service social ne peut exister sans la vocation. Son but est de réintégrer l'individu ou la famille dans la société en utilisant différentes ressources, dont la principale est la compétence du travailleur social. (Groulx, 1993, p. 42)

Une telle compétence résidait à la fois en des connaissances et en des attitudes spécifiques comme un « équilibre de jugement et d'émotion, intérêt réel pour les malheureux et foi dans l'individu et la famille»(Groulx, 1993, p. 42). L'embauche et la sélection des auxiliaires sociales se réalisaient ainsi sur la base de qualités personnelles et d'une forme de foi individuelle dans le métier, plutôt que sur des compétences à caractère plus professionnel acquises dans le cadre d'un processus de formation au travail social. En réalité, au-delà de l'apprentissage plus technique d'un processus d'enquête rationnel (Richmond, 1917), des caractéristiques individuelles, généralement attribuées au sexe féminin, étaient recherchées chez les candidates, notamment des « vertus oblatives d'accueil, de générosité, de sacrifice, de dévouement, de sociabilité chaleureuse et de compréhension affective "(Lord, 1948). En dépit de l'importance affirmée de la relation et de la méthode comme principaux facteurs de réussite des interventions sociales, il apparaît cependant que ceux-ci ne découlent pas d'un même registre d'apprentissage, ainsi que le souligne Du Ranquet (1991, p. 3) : « le travail social se définit par la méthode qui s'appuie sur des connaissances et la relation qui s'appuie sur des valeurs. "

L'adoption d'un discours laïque et rationnel (Mayer et Groulx, 1987) et de méthodes d'intervention appuyées sur des bases théoriques plus ou moins propres à la profession, comme principalement la psychologie et la sociologie, caractérisera les efforts du travail social pour se distancier de ses origines premières au cours des années 1950 (Lecomte, 2000). L'idée derrière le recours aux courants théoriques psychologiques et sociologiques était de participer à la construction d'une base argumentaire plus solide et plus légitime aux yeux des acteurs de la profession que les fondements religieux d'origine. Il demeure incontestable que ces principales disciplines mères ont eu des impacts importants sur la profession, tant sur le plan théorique, comme façons de définir et 
"...tout se passe en fin de compte comme si la question de l'identité professionnelle devait être assumée et satisfaite par chaque travailleuse on travailleur social de manière isolée..." de concevoir le travail social, que sur un plan plus méthodologique, comme manières de pratiquer l'intervention. Néanmoins, malgré le fait que sur le plan de l'intervention de nombreux modèles de pratique aient été créés en appui sur ces fondements théoriques, il appert que d'un point de vue conceptuel aucune théorie unique et totalement intégrée et aucun corpus de connaissances communes et propres au travail social ne sont issus du recours aux théories (Deslauriers et Hurtubise, 2000); ce serait en réalité surtout autour de "morceaux de savoirs empruntés généralement à la psychologie que s'est principalement constitué un corpus de référence " (p. 52) pour le travail social individuel. Certains avancent que, de par ses origines multiples, il s'avère difficile pour la profession d'intégrer les diverses traditions à partir d'un savoir qui serait propre à l'ensemble du travail social (Ion, 2006). Cette diversité est d'ailleurs facilement repérable par l'affirmation du caractère pragmatiste et éclectique de la profession. La grande diversité théorique et méthodologique typique du travail social fait même pour plusieurs (Dorvil et Mayer, 2001; Groulx, 1993; Hurtubise et Deslauriers, 2003; Molgat, 2007; Mayer, et collab., 2000) office de base épistémologique à la profession. En appui sur cette posture épistémologique, tout se passe en fin de compte comme si la question de l'identité professionnelle devait être assumée et satisfaite par chaque travailleuse ou travailleur social de manière isolée, en fonction des éléments personnels, organisationnels ou professionnels qui l'encadrent. On devine bien les racines d'un malaise derrière ce type de stratégie. Que "font " dans les faits, les travailleurs sociaux? Quelles sont les productions concrètes du travail social? Devant la carence d'arguments soutenant ce que les travailleurs sociaux " font » concrètement, le repli sur l'identité, sur ce que les travailleurs sociaux "sont ", apparait intelligible.

Si la tentative de disciplinarisation du travail social s'est fondée sur la recherche de savoirs scientifiques propres à la profession, il appert toutefois que les savoirs et les méthodologies des deux disciplines mères ont contribué à normer le travail social, sans chercher à conceptualiser la pratique des travailleurs sociaux telle qu'elle est réalisée dans les faits. Les fondements du travail social ne semblent d'ailleurs pas à la base avoir cherché à 
"Ni les fondements religieux ni les fondements théoriques n'ont fourni de cadre référentiel ou de corpus de savoirs qui soient spécifiques au travail social. » expliquer le travail social ou à expliquer ce que les travailleurs sociaux accomplissent réellement au quotidien. Les fondements axiologiques, institutionnels et théoriques ont surtout visé à fournir aux travailleurs sociaux de bonnes raisons d'agir comme ils le font et ont eu pour principaux objectifs de justifier leur rôle au sein de la société. Ni les fondements religieux ni les fondements théoriques n'ont fourni de cadre référentiel ou de corpus de savoirs qui soient spécifiques au travail social. Dans un cas comme dans l'autre, la nature des discours est demeurée avant tout normative et le rapport à la profession est resté le même :prescrire les bonnes pratiques des travailleurs sociaux. La référence à la théorie par les travailleurs sociaux s'est alors surtout avérée utile pour justifier la nécessité externe d'agir plutôt que pour soutenir leur décision d'agir et d'argumenter sur les raisons pour lesquelles ils interviennent dans les faits (Zúñiga, 1993); la théorie est donc venue appuyer surtout une logique de légitimation plutôt qu'une logique d'objectivation. Qui plus est, le fait pour les travailleurs sociaux de devoir rechercher le sens de leur action professionnelle à partir de travaux théoriques que le travail social ne produit pas lui-même crée chez eux une tension supplémentaire (Dubet, 2002). Le réflexe répandu du refuge dans le vécu de la pratique empirique, dans l'essence du "vrai » travail social, ne résoudrait par ailleurs pas le malaise, car le vécu, tant qu'il n'est pas traduit et expliqué à partir d'une base théorique, ne renseigne pas sur la signification de l'agir professionnel (Dubet, 2002).

Les transformations étatiques et le recours aux théories des disciplines mères du travail social ont donc été déterminants dans sa professionnalisation, mais insuffisants pour lui permettre d'accéder au statut de discipline clairement et indiscutablement scientifique, et ce, malgré les efforts pour se dégager du caractère vocationnel. L'effort de rationalisation de la profession se heurte en effet à un obstacle majeur, celui de la résistance de la forme de connaissance classique du travail social dans un contexte où la profession est appelée à se poser comme scientifique. Une tension émerge ainsi d'une volonté à unifier à une exigence de rigueur une profession qui tend à rester centrée sur les valeurs. Devant cette difficile harmonisation des fondements religieux- 
humanistes et axiologico-théoriques du travail social, il apparait compréhensible que la spécificité de la profession ne s'appréhende ni ne se conçoive encore si aisément aujourd'hui.

\section{Conclusion}

«...l'une des voies qui pourraient permettre de considérer autrement le cour de la profession serait de chercher à conceptualiser ces savoirs..."
Les approches axiologiques et normatives qui ont historiquement veillé à orienter le travail social ne semblent en fin de compte pas être parvenues à réduire le sentiment de non-reconnaissance que vivent bon nombre des acteurs de la profession. Il va sans dire que la complexité de sa dimension relationnelle rend ardue toute tentative d'élucidation de l'ensemble de ses composantes. Son analyse constitue en effet un vaste défi, puisque la présence d'interactions multiples (Mayen, 2007), l'existence d'au moins deux acteurs (Goffman, 1974), les déterminants organisationnels pesant sur la relation (Soulet, 1997) ou encore la nature du service à rendre (Boujut, 2005) ajoutent un obstacle supplémentaire à l'objectivation des savoirs spécifiques à l'exercice d'une profession aussi hautement relationnelle que le travail social. Pourtant, l'une des voies qui pourraient permettre de considérer autrement le cœur de la profession serait de chercher à conceptualiser ces savoirs qui s'opérationnalisent au sein même des interactions, toujours situées, qui ont cours entre un travailleur social et un usager.

Au-delà des valeurs humanistes et sociales dont l'intervention sociale se veut le reflet et de la maitrise de différents modèles ou approches d'intervention par le travailleur social, il demeure que la pratique professionnelle de tout travailleur social permet l'accomplissement du travail social. C'est donc que certains axes fondamentaux traversent l'ensemble des pratiques de la profession. Indépendamment du milieu de travail ou des allégeances de chacun, toute relation s'établissant entre un travailleur social et un usager recèle l'engagement de satisfaire une double demande, individuelle et sociale (Autès, 1998), et l'intention de transformer un rapport entre un usager en souffrance et une norme toujours socialement construite. C'est ainsi que par la relation, le travail du travailleur social consiste à rattacher symboliquement les individus 
à la société en donnant du sens au rapport qui s'établit entre leurs difficultés, toujours singulières, et les problèmes sociaux et leur réponse sociale sous-jacents. L'action du travail social est en ce sens une action essentiellement médiatrice. C'est donc précisément cette action de médiation, ce travail symbolique de production de sens pour l'individu, d'objectivation de sa difficulté en regard d'une norme sociale, qui constitue la spécificité du travail social (Chouinard, Couturier et Lenoir, 2009).

À la lumière de ces éléments, le fait de chercher à mettre à jour la structure médiatrice fondamentale de l'intervention sociale pourrait offrir l'opportunité d'élucider les référents professionnels du travail social permettant par voie de conséquence d'appuyer l'énonciation, l'analyse et l'apprentissage de l'une des dimensions les plus cruciales de la profession : la relation.

\section{Bibliographie}

ASSOCIATION CANADIENNE DES TRAVAILLEURS SOCIAUX (2012). Orientations stratégiques de l'ACTS 2012-2015, Ottawa, ACTS., 7 p.

AUTÈS, Michel (1998). "Le travail social ou la relation de service sans service ", Lien social et politiques-RIAC, No 40, p. 47-53.

BOUJUT, Stéphanie (2005). «Le travail social comme relation de service ou la gestion des émotions comme compétence professionnelle : normes, déviances, réactions sociales sous le regard de jeunes sociologues français ", Déviance et société, Vol. 29, No 2, p. 141-153.

CAREY-BÉLANGER, Elaine (1979). «Les débuts et le développement de la corporation (19601979) ", Intervention, No 56, p. 5-25.

CHOUINARD, Isabelle, Yves COUTURIER et Yves LENOIR (2009). "Pratique de médiation ou pratique médiatrice? La médiation comme cadre d'analyse de la pratique professionnelle des travailleurs sociaux ", Nouvelles pratiques sociales, Vol. 21, No 2, p. 31-45.

COUTURIER, Yves, et Isabelle CHOUINARD (2008). " La relation est-elle soluble dans la didactique? La relationnalité dans les métiers relationnels comme objet d'une didactique des savoirs professionnels ", dans Yves Lenoir et Pierre Pastré (dirs.). Didactique professionnelle et didactiques disciplinaires. Un enjeu pour la professionnalisation des enseignants. Toulouse, Octarès Éditions, p. 213-223.

DE MONTMOLLIN, Maurice (1986). L'ergonomie, Paris, Éditions La Découverte, 126 p.

DESLAURIERS, Jean-Pierre, et Yves HURTUBISE (dirs.) (2000). Introduction au travail social, SteFoy, Presses de l'Université Laval, 426 p.

DORVIL, Henri, et Robert MAYER (dirs.) (2001). Problèmes sociaux, Tome 1-Théories et méthodologies, Ste-Foy, Presse de l’Université du Québec, 622 p. 
DUBET, François (2002). Le déclin de l'institution, Paris, Seuil, 428 p.

DU RANQUET, Mathilde (1991). Les approches en service social, Montréal, Edisem, 222 p.

GARNIER, Jean-François (2000). «Aux fondements du travail social», dans «Travail social :l'individu, le groupe, le collectif ", Informations sociales, $\mathrm{N}^{\circ} 83, \mathrm{p} .14-25$.

GIULIANI, Frédérique (2007). "La procédure de l'entretien individualisé dans le travail d'accompagnement : quand usagers et intervenants sociaux ont à organiser l'expérience de situations sans qualité », dans Isabelle Astier et Nicolas Duvoux (dirs.), La société biographique : une injonction à vivre dignement, Paris, L'Harmattan, p. 195-212.

GOFFMAN, Erving (1974). Les rites d'interactions, Paris, Les éditions de Minuit, 240 p.

GROULX, Lionel-Henri (1993). Le travail social : analyse et évolution, débats et enjeux, Québec, Éditions Agence d'ARC, $297 \mathrm{p}$.

HURTUBISE,Yves, et Jean-Pierre Deslauriers (dirs.) (2003). Introduction au travail social. Méthodologies et pratiques nord-américaines, Ste-Foy, Presses de l'Université Laval, $255 \mathrm{p}$.

ION, Jacques (1998). Le travail social au singulier. La fin du travail social?, Paris, Dunod, $160 \mathrm{p}$.

ION, Jacques (dir.) (2005). Le travail social en débat(s), Paris, La Découverte, 267 p.

ION, Jacques, et Bertrand RAVON (1984). Les travailleurs sociaux, Paris, La Découverte, 125 p.

JENNISSEN, Therese, et Colleen LUNDY (s.d.). Garder la justice sociale dans notre mire : 80 années de développement pour l'ACTS, réf. du 25 mai 2013, http://www.casw-acts.ca/sites/default/files/ attachements/L\%27Historique\%20de\%201\%27ACTS.pdf

LECOMTE, Roland (2000). "Fondements théoriques et identité professionnelle en service social ", Politiques sociales, № 1-2, p. 12-24.

LORD, L. (1948). " Avantages du Service Social catholique ", La Presse, 7 décembre 1948.

MAYEN, Patrick (2007). "Quelques repères pour analyser les situations dans lesquelles le travail consiste à agir pour et avec un autre ", Cahiers du CREN, No 4, p. 51-64.

MAYER, Robert (2002). Évolution des pratiques en service social, Boucherville, Gaétan Morin éditeur, $489 \mathrm{p}$.

MAYER, Robert, et Lionel-Henri GROULX (1987). Synthèse critique de la littérature sur l'évolution des services sociaux au Québec depuis 1960, Gouvernement du Québec, Commission d'enquête sur les services de santé et les services sociaux, $512 \mathrm{p}$.

MAYER, Robert, et collab. (dir.) (2000). Méthodes de recherche et intervention sociale, Boucherville, Gaëtan Morin, 410 p.

MOLGAT, Marc (2000). « Définir le travail social... », dans Jean-Pierre Deslauriers etYves Hurtubise (dirs.), Introduction au travail social, Ste-Foy, Presses de l'Université Laval, p. 19-40.

PELCHAT, Yolande, et collab. (2004). La pratique de l'intervention sociale et psychosociale en CLSC, Rapport de recherche. Équipe RIPOST, CLSC-CHSLD Haute-Ville-Des-Rivières, Québec, $76 \mathrm{p}$.

RICHMOND, Mary E. (1917). Social Diagnosic, New-York, Sage Fundation, 516 p.

SOULET, Marc Henry (1997). Petit précis de grammaire indigène du travail social : Règles, principes et paradoxes de l'intervention sociale au quotidien, Fribourg, Éditions Universitaires Fribourg Suisse, $268 \mathrm{p}$.

STEPHENSEN, Marylee, et collab. (2000). Le travail social au Canada : une profession essentielle Volume 1 : Rapport final. Développement des ressources humaines du Canada. 
VILBROD, Alain (dir.) (2003). L'identité incertaine des travailleurs sociaux, Paris, L'Harmattan, 430 p. VILLATE, Robert, Catherine TEIGER et Sandrine CAROLY-FLAGEUL (2004). « Le travail de médiation et d'intervention sociale ", dans Pierre Falzon (dir.), Traité d'ergonomie, Paris, PUF, p. 582-601

ZÚÑIGA, Ricardo (1993). "La théorie et la construction des convictions en travail social ", Service social, Vol. 42, $\mathrm{N}^{\circ} 3$, p. 33-54. 